\title{
Review Article \\ Interluekin-35 in Asthma and Its Potential as an Effective Therapeutic Agent
}

\author{
Peng Gao, Zhenzhong Su, Xuejiao Lv, and Jie Zhang \\ Department of Respiratory Medicine, The Second Affiliated Hospital of Jilin University, Changchun, China \\ Correspondence should be addressed to Jie Zhang; doctorzhangj@sina.com
}

Received 13 February 2017; Accepted 26 March 2017; Published 3 May 2017

Academic Editor: Tânia Silvia Fröde

Copyright @ 2017 Peng Gao et al. This is an open access article distributed under the Creative Commons Attribution License, which permits unrestricted use, distribution, and reproduction in any medium, provided the original work is properly cited.

\begin{abstract}
Interleukin- (IL-) 35 is a member of the IL-12 cytokine family and a heterodimeric protein formed by Epstein-Barr-induced gene 3 (EBI3) and IL-12p35. Emerging evidence shows that IL-35 is a key player in the regulation of cellular communication, differentiation, and inflammation. Altered IL-35 expression has been found in disease conditions such as cancer, rheumatoid arthritis, and, more recently, asthma. In cancer, IL-35 is involved in the regulation of tumorigenesis, cancer progression, and metastasis. In rheumatoid arthritis, IL-35 acts as a negative regulator of inflammation. Similarly, IL-35 also appears to suppress allergic inflammation in asthma. In an in vivo murine model of asthma, transfer of adenovirus-mediated IL-35 markedly reduced the degree of airway hyperresponsiveness (AHR) and inflammatory cell infiltration. Many studies have shown the involvement of IL-35 in a number of aspects of allergic inflammation, such as eosinophil and neutrophil recruitment as well as inhibition of inflammatory mediators of the Th2 subtype. However, the exact molecular mechanisms underlying the role of IL-35 in human asthma have yet to be fully elucidated. This review describes the current evidence regarding the role of IL-35 in the pathophysiology of asthma and evaluates the potential of IL-35 as a biomarker for airway inflammation and a therapeutic target for the treatment of asthma.
\end{abstract}

\section{Introduction}

Interleukin- (IL-) 35 is a member of the IL-12 cytokine family $[1,2]$ and was first described almost two decades ago as a heterodimer formed by the subunits Epstein-Barr-induced gene 3 (EBI3) and IL-12p35. It was found in the trophoblast component of the human placenta and suggested to be an important immunoregulator already upon discovery [3]. IL-35 induces the generation of a unique class of IL-35producing regulatory $\mathrm{T}$ cells (Tregs) that are distinctly different from the other transforming growth factor beta(TGF- $\beta$-) or IL-10-induced Tregs. These cells are called IL35 -producing iTregs (iTr35 cells) $[1,4]$. IL-35 secreted by iTr35 cells in turn induces the generation of even more iTr35 cells, and thus, IL-35 and iTr35 cells create a positive feedback loop together [1].

The IL-35 receptors on the cell surface can be either homodimers of IL-12R $\beta_{2}$ or gp130 or a heterodimer of IL$12 \mathrm{R} \beta_{2}$ :gp130 [2]. Binding of IL-35 to its receptors leads to activation of the JAK-STAT pathway, which requires the transcription factors STAT1 and STAT4 to form a unique heterodimer [2] and induces immunosuppression, IL-35 production, and conversion of naive $\mathrm{T}$ cells into iTr 35 cells. The heterodimeric IL-12R $\beta_{2}$ :gp130 receptor is required for the maximal effects to be achieved [2] (Figure 1). While the gp130 subunit is widely expressed in many different cell types, the IL-12R $\beta_{2}$ subunit is mainly found on activated T cells of the Th1 subtype and natural killer cells [5]. In most resting T cells, IL-12R $\beta_{2}$ is almost undetectable. However, exposure to IL-2, IL-12, IL-27, interferon- (IFN-) $\gamma$, and tumor-necrosis factor (TNF) can rapidly increase the expression of IL-12R $\beta_{2}$ [2]. Expression of IL-12R $\beta_{2}$ has also been shown on other immune cells, such as dendritic cells [6].

IL-35 is produced by a wide range of tissues and cell types, including monocytes, T cells, B cells, Tregs, and tumor cells under resting conditions $[7,8]$. Upon Toll-like receptor stimulation, IL-35 subunits are also produced by macrophages and dendritic cells [9]. Recently, Guttek and Reinhold 


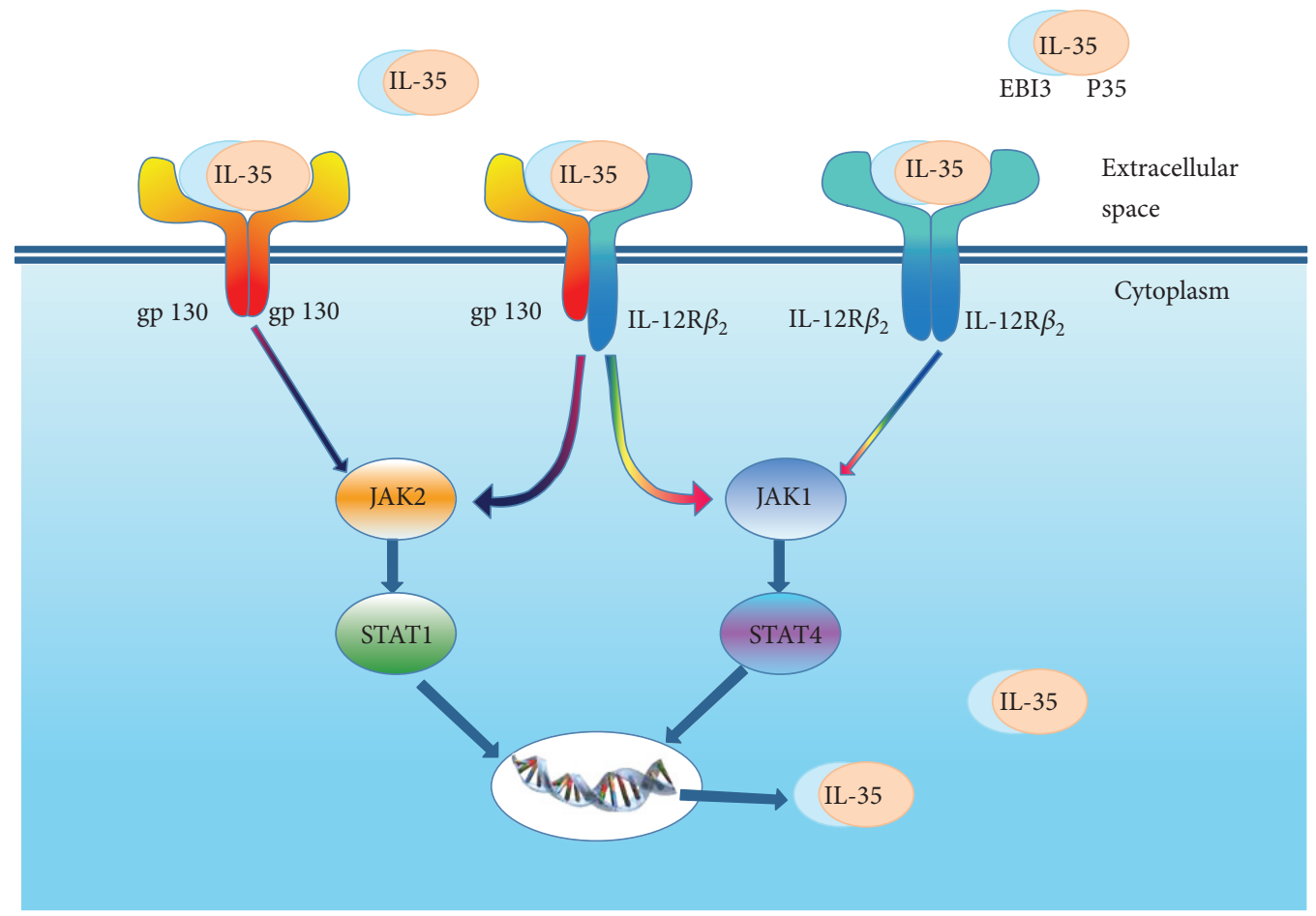

FIGURE 1: The structure of IL-35 and the activation of the JAK-STAT signaling pathway.

showed that both resting and stimulated $\mathrm{CD} 4{ }^{+} \mathrm{CD} 25^{+} \mathrm{T}$ cells from humans secrete high amounts of IL-35, whereas peripheral pan $\mathrm{T}$ cells or $\mathrm{CD} 4^{+}, \mathrm{CD} 8^{+}$, and $\mathrm{CD} 4^{+} \mathrm{CD} 25^{-} \mathrm{T}$ cells produce IL-35 only upon stimulation [10].

The primary physiological role of IL-35 is to regulate T cell homeostasis. It inhibits T helper (Th) 2 and Th17 polarization. Niedbala et al. [7] demonstrated that IL-35 inhibits Th17 cell differentiation in vitro and suppresses IL-17 production in vivo in mice. Similar conclusions were reached by Liu et al. [11] who demonstrated increased $\mathrm{T}$ cell production of IL-17 in the peripheral lymphoid organs of EBI3deficient mice, supporting an inhibitory role for EBI3 against Th17 polarization. Spleen cells from mice deficient in the IL-35 subunit EBI3 display increased expression of IL-17 and IL-22 as well as retinoid-related orphan receptor $\gamma \mathrm{t}$ $(\mathrm{ROR} \gamma \mathrm{t})$, which is the key transcription factor regulating Th17 cell differentiation [12]. IL-35 secreted by inducible costimulator-positive Tregs has been found to suppress Th17 activity and thereby reduce the degree of neutrophilia and airway hyperresponsiveness (AHR) found in the airways of mice sensitized and challenged with ovalbumin (OVA) [13]. IL-35 also expands Tregs, specifically iTr35 cells, both in vitro and in vivo $[1,7]$. However, the results have not been unanimous, as one study also showed a reduction in the Treg response induced by IL-35 [11].

We searched PubMed for papers published between the periods 2000 and 2017 including the key word "IL-35". Relevant papers were included after manual selection based on the abstracts and discussion within the research group. The present review summarizes the published literature on the functions and mechanisms of IL-35 in both humans and experimental models, explores its role in the pathophysiology of asthma, and discusses the potential of IL-35-based therapeutic strategies against asthma.

1.1. Expression of IL-35 in Human Diseases. The body of literature studying the effects of IL-35 has expanded rapidly in recent years. Such increased interest might be due to the broad range of biological functions associated with IL-35, including cell differentiation, tumor progression, and immunoregulation $[1,14,15]$. Moreover, IL-35 has been found to be involved in the physiopathology of a range of different diseases (Table 1). The reported normal levels of IL-35 are however highly variable in literature. This might be due to the different ELISA kits and standards used.

To date, many aspects of inflammatory and autoimmune disease have been linked to IL-35 function, such as suppression of $\mathrm{CD} 4^{+}$effector T cells $[1,7,16,17]$, Th17 cell development and differentiation [7], and attenuation of allergen-specific Th2 responses and production of Th2 cytokines [18]. Initially, IL-35 was thought to have anti-inflammatory effects on immune-mediated inflammatory diseases through the suppression of $\mathrm{T}$ cell proliferation and effector functions. However, a recently published study demonstrated a paradoxal proinflammatory effect of IL-35. In mice with collageninduced arthritis (CIA), treatment with IL-35 gene transfer actually exacerbated arthritis symptoms and at the same time increased the Th17 cell/Treg ratio in the spleen [19]. These contradictory results suggest that the in vivo functions of IL-35 are complex and that additional studies are required to truly elucidate the roles of IL-35 in health and disease.

Aberrant expression of extracellular IL-35 has been detected in the inflammatory setting both in animal models and human serum in vivo. Remarkably increased expression 


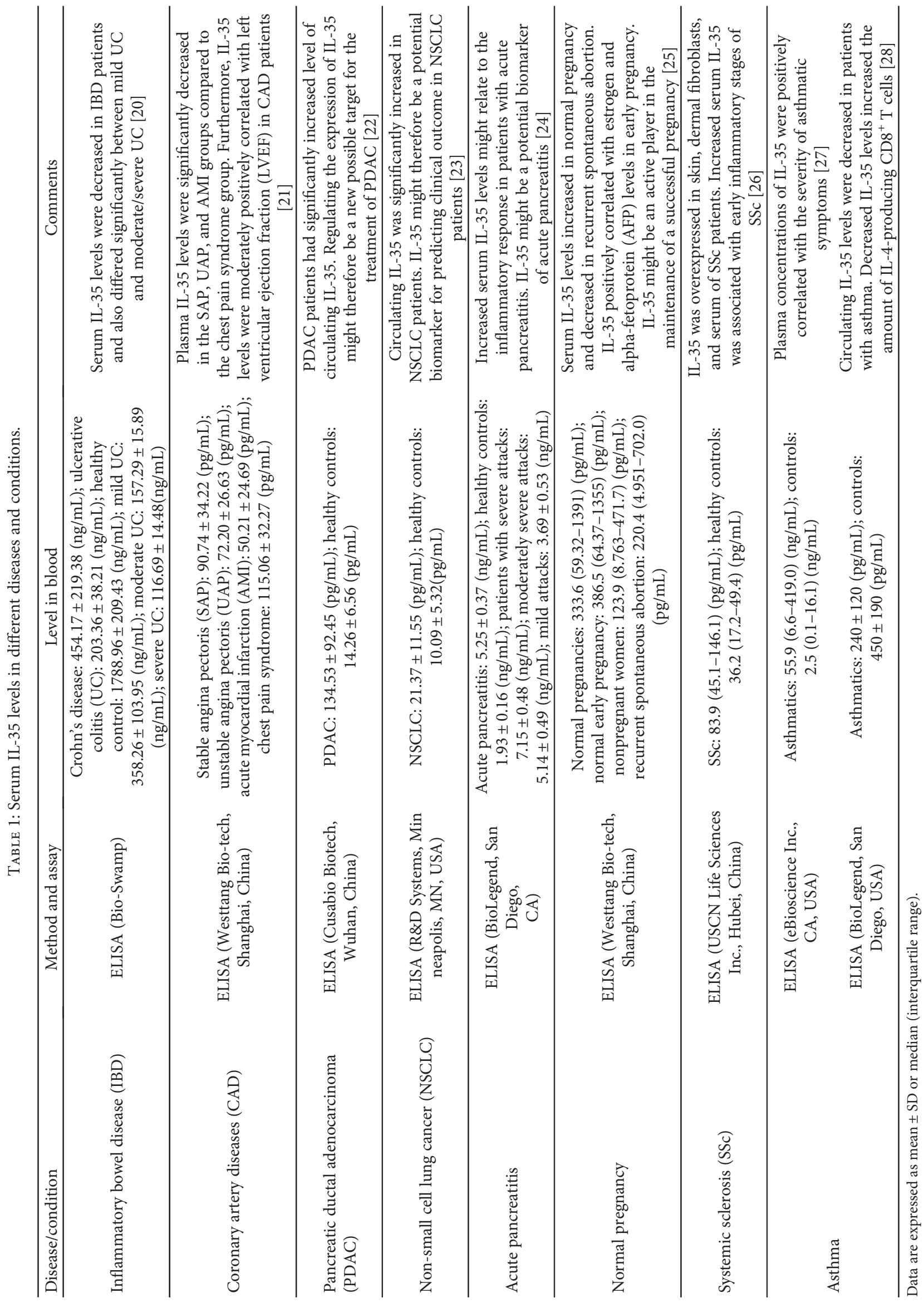


of EBI3 and p35 was detected in intestinal epithelial cells from wild-type mice by qPCR. Intestines of EBI3-deficient mice showed increased degree of colitis compared to those of control mice, while treatment with exogenous recombinant IL-35 decreased intestinal inflammation by suppression of Th1- and Th17-dependent inflammatory responses [9]. In humans, patients with ulcerative colitis (UC) and Crohn's disease (CD) were found to have significantly lower levels of serum IL-35 than healthy individuals, and the cytokine level was inversely correlated with UC activity. The gene and protein expression levels of EBI3 and p35, which are the two subunits of IL-35, were significantly higher in the intestinal mucosa of patients with inflammatory bowel disease (IBD) compared to that of healthy controls [20]. Atherosclerosis and coronary artery disease (CAD) have long been thought to be caused by disrupted lipid metabolism but have gained wide recognition as inflammatory conditions in recent years. It has been shown that plasma IL-35 levels are significantly lower in patients with stable and unstable angina pectoris, as well as acute myocardial infarction compared with the levels in patients with chest pain syndrome. In CAD patients, IL-35 levels were negatively correlated with the degree of heart failure, defined by a decreased left ventricular ejection fraction (LVEF) [21].

1.2. The Role of IL-35 in Human Asthma. Asthma is a chronic inflammatory condition of the respiratory system characterized by abnormal $\mathrm{T}$ cell responses [29]. The asthmatic inflammation is finely orchestrated by $\mathrm{CD} 4^{+} \mathrm{T}$ lymphocytes, including Th1, Th2, and Th17 cells [30]. Airway inflammation leads to increased bronchial contractions termed airway hyperresponsiveness (AHR) and asthmatic symptoms such as wheezing, shortness of breath, and chest tightness [31].

Recent research has shown that asthma is a heterogeneous disease, and much effort has been put into subtyping the different phenotypes of asthma. A common method of subtyping asthma is based on the presence of different inflammatory cells in induced sputum, with a classification into four different groups: neutrophilic asthma, eosinophilic asthma, mixed granulocytic asthma, and paucigranulocytic asthma. Each subtype has a distinct mechanism and responds to therapy differentially [32-34]. Recognition that asthma is not one homogenous disease and that different subtypes of asthma exist is crucial for understanding the underlying disease processes and the development of the so called "personalized medicine." Classic eosinophilic asthma is the best-characterized subtype and responds well to corticosteroids, which are the first-line therapy against asthma used in the clinic today. The mechanisms underlying eosinophilic asthma primarily involve allergen-induced activation of Th2 pathways and release of Th2 cytokines, such as IL-4, IL-5, IL-9, and IL-13 [35]. Neutrophilic asthma, on the other hand, is driven by the activation of the innate immune system including Toll-like receptors (TLRs) and NLRP3 inflammasome by infections and pollutants [36, 37]. This subtype of asthma is much less well characterized and notoriously resistant to corticosteroid treatment. The innate immune system including Toll-like receptors (TLRs) and NLRP3 inflammasome has been shown to be involved in neutrophilic asthma [36, 37]. More recently, Th17 cells, which produce IL-17 and IL-22 and mediate neutrophil recruitment [38], have also been shown to have an influential role [39].

IL-35 may be differentially involved in the pathogenesis of the different phenotypes of asthma. IL-17, which can be suppressed by IL-35, is involved in both neutrophilic and eosinophilic asthma. IL-17 produced by Th17 cells or macrophages not only promotes the infiltration of neutrophils into the lung but also, as suggested by recent evidence, can contribute to the development of allergic eosinophilic asthma [40, 41] and act synergistically with Th2 cytokines to promote AHR [39, 40, 42]. Tregs can secrete IL-35 [3], which in turn suppresses Th1, Th2, and Th17 effector responses to keep inflammatory responses in check $[43,44]$ (Figure 2). Defective Treg regulation is associated with the pathogenesis of both Th2- and Th1-mediated inflammatory diseases [45, 46], including asthma, which is a classic Th2-mediated inflammatory disease $[47,48]$. Interestingly, decreased numbers of Tregs were also detected in the lungs of patients with tobacco smoking-induced emphysema [49], suggesting that Tregs regulate not only asthmatic inflammation but also emphysemarelated inflammation in the lungs. Rats exposed to cigarette smoke also had significantly lower IL-35 concentrations in their bronchoalveolar lavage fluid (BALF) compared to control rats [50].

Despite the good theoretical possibility, the role of IL-35 in asthma is still not well established. Wong et al. found significantly elevated IL-35 levels in patients with allergic asthma compared to those in nonallergic controls by evaluating plasma concentrations of IL-35 using an enzyme-linked immunosorbent assay (ELISA). Plasma concentrations of IL-35 were clearly positively correlated with the severity of asthmatic symptoms [27]. However, another study by Wang et al. reported completely opposite results suggesting instead that levels of circulating IL-35 are decreased in patients with asthma, and that decreased IL-35 levels increase the amount of IL-4-producing $\mathrm{CD}^{+} \mathrm{T}$ cells [28]. These contradictions in experimental results might be explained by the heterogeneity of airway inflammation in asthma and differences in the subtypes of asthma that were studied. Thus, additional studies with carefully phenotyped asthmatic patients are required to fully characterize the expression of IL-35 in the blood of asthmatic patients.

1.3. IL-35 in Experimental Models of Asthma. Increasing numbers of studies suggest that IL-35 can suppress airway inflammation and AHR in experimental models of asthma. Mice deficient in the IL-35 subunit EBI3 or p53 displayed higher degrees of airway inflammation, higher levels of IL-17, and sustained AHR compared to wild-type mice after the treatment with an OVA-lipopolysaccharide (LPS) sensitization and challenge protocol, suggesting that IL-35 could suppress AHR [13]. In the mouse OVA model of asthma, treatment with either adenovirus-mediated IL-35 [51] or recombinant IL-35 [52] suppressed both AHR and the degree of allergic inflammation. Although the OVA model of asthma is considered a "classic" model, it is also deemed to be too "artificial" and lacks the features of chronic airway 


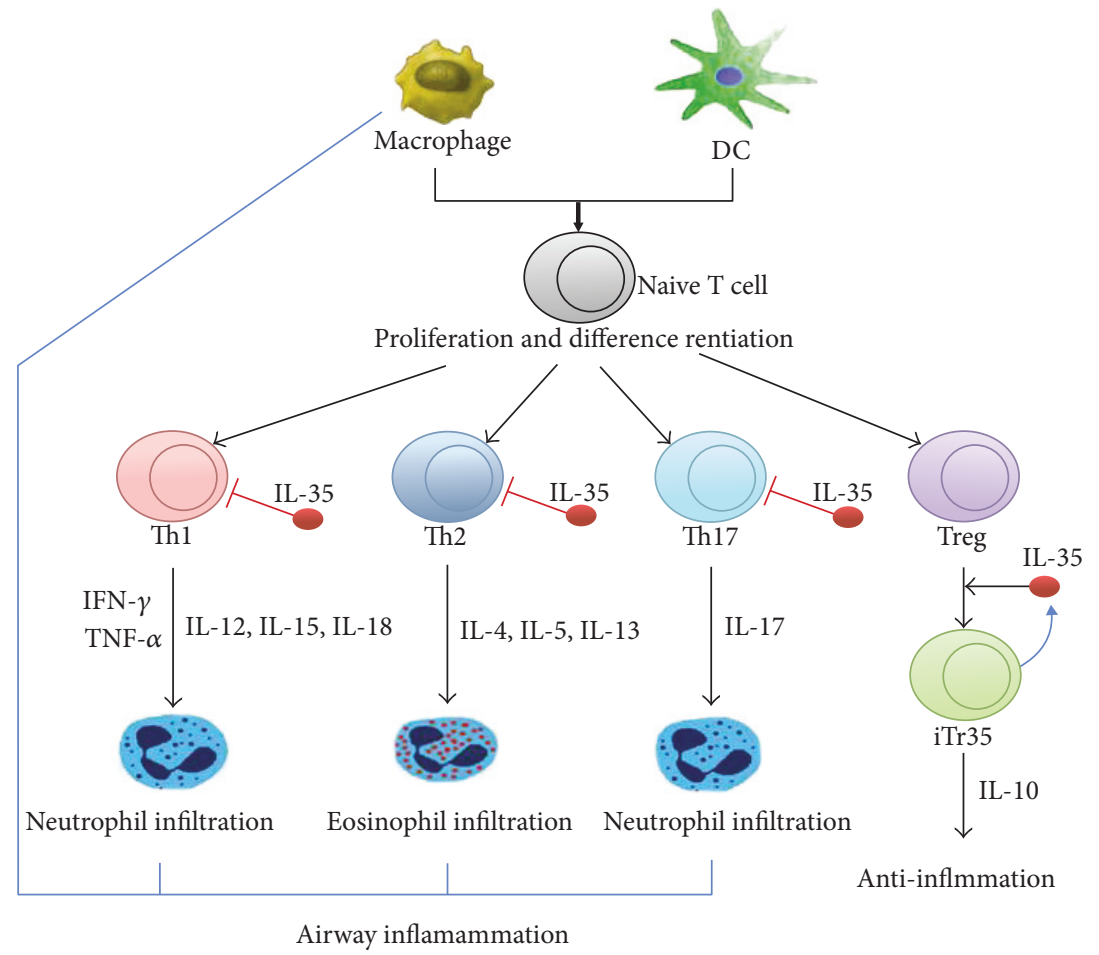

FIgURe 2: Function of IL-35 in asthma. The black arrow indicates positive effects, and the T-shaped end indicates negative effects. DC: dendritic cell; IL: interleukin; Th: helper T cell. Adapted from Pope and Shahrara [4].

inflammation. The effects of anti-IL-35 therapy has therefore also been studied in a model using the "real-life" allergen house dust mite (HDM). Mice with glucocorticoid-sensitive eosinophilic airway inflammation induced by a HDM allergenspecific memory/effector Th2 cell line that is treated with pVAX-IL-35 DNA via an intranasal route showed decreased allergen-specific airway inflammation, while intramuscular injection of pVAX-IL-35 suppressed levels of circulating allergen-specific and total IgE over an extended period of time [18]. Taken together, these studies suggest that IL-35 may be a new effective therapeutic agent for the treatment of allergic asthma.

Several different mechanisms have been suggested to mediate the effects of IL-35. IL-35 affects a wide range of different inflammatory cells. It is likely that IL-35 does not exert its anti-inflammatory effect via one single mechanism, but rather simultaneously affects a number of different inflammatory pathways.

1.3.1. Th Cells. IL-35 induces differentiation of $\mathrm{CD} 4^{+}$effector $\mathrm{T}$ cells into a specific subset of Tregs called iTr35 cells. These cells express IL-35, but not Foxp3, TGF- $\beta$, or IL-10, and can suppress inflammation in animal models of experimental autoimmune encephalomyelitis (EAE) and IBD [1].

In vitro, IL-35 has different effects on effector $\mathrm{T}$ cells dependent on the inflammatory condition. Under acute inflammatory conditions such as acute infections, IL-35 induces expansion of both $\mathrm{CD} 4^{+} \mathrm{CD} 25^{+}$and $\mathrm{CD} 4^{+} \mathrm{CD} 25^{-} \mathrm{T}$ cells, but under conditions mimicking chronic infection/ inflammation, IL-35 instead suppresses $\mathrm{CD} 4^{+} \mathrm{CD} 25^{-}$effector cell proliferation and Th17 cell differentiation [11]. Spleen cells from mice deficient in the IL-35 subunit EBI3 display increased expressions of IL-17 as well as retinoid-related orphan receptor $\gamma \mathrm{t}(\mathrm{ROR} \gamma \mathrm{t})$, which is the key transcription factor regulating Th17 cell differentiation [12].

Th17 levels are found to be elevated in the sputum of asthmatic patients and to correlate with their degree of airway hyperreactivity [41]. In mice, IL-35 effectively suppresses IL-17 production, while at the same time enhancing interferon-gamma synthesis, and thereby attenuates established CIA in vivo [7]. Treatment with exogenous IL-35 inhibits the development of inflammation in mice with CIA via suppression of Th1 and Th17 cells and production of IL- 10 by $\mathrm{CD} 25^{-} \mathrm{CD} 39^{+} \mathrm{CD} 4^{+} \mathrm{T}$ cells [16]. In addition, IL-35 attenuates Th2-type airway inflammation induced by HDM allergen-specific memory/effector Th2 cells [18].

1.3.2. Dendritic Cells. IL-35 has been shown to suppress dendritic cell (DC) migration to the lung and draining of mediastinal lymph nodes (mLNs) [52]. In a murine OVA model of asthma, IL-35 interrupts the conversion of the recruited monocytes to inflammatory DCs and at the same time attenuates the accumulation of migratory $\mathrm{CD} 11 \mathrm{~b}^{+} \mathrm{CD} 103^{-} \mathrm{DCs}$ in the mLNs and lungs. Mice that received IL-35 on days 1 and 7 during OVA sensitization had a decreased percentage and absolute number of $\mathrm{CD} 11 \mathrm{~b}^{+} \mathrm{CD} 103^{-}$DCs in the mLNs [52].

1.3.3. Eosinophils and Neutrophils. Airway inflammation in the murine OVA model is predominately eosinophilic and accompanied by large amounts of Th2 cytokines, but small amounts of neutrophils and mononuclear cells can also be found. Intraperitoneal injection of IL-35 during sensitization 
significantly suppressed the inflammatory response, decreasing the number of infiltrating eosinophils and Th2 cytokine levels in BALF and lung tissue [52]. Moreover, in a model of airway inflammation induced by a HDM allergenspecific Th2 cell line, local administration of a plasmid that enhances IL-35 production significantly decreased the levels of eosinophilia, neutrophilia, total IgE, and the Th2 cytokine IL-4 [18]. Moreover, OVA-/LPS-induced neutrophilia and AHR in mice were inhibited by IL-35 via suppression of Th17 activity [13].

1.3.4. B Cells. IL-35 is also known to affect antibody-secreting cells (ASCs), more specifically B cells. B cells boost immunity through the production of antibodies, but the subset of regulatory B cells (Bregs) can also suppress immunity through the production of IL-10, TGF-beta, and IL-35. Bregs, a set of ASCs that have been shown to play an interesting immunomodulatory role in allergic airway inflammation, are distinct [53]. They share a surface $\operatorname{IgM}^{+}$CD138hi CD44hi CD69 ${ }^{+}$ $\mathrm{TACI}^{+} \mathrm{C} \mathrm{XCR}^{+}$CD1dint TIM-lint $\mathrm{MHC}^{+} \mathrm{II}^{+} \mathrm{CD}^{+} 0^{+}$ $\mathrm{CD}^{+} 6^{+}$phenotype and express the transcription factors Blimp1 and IRF4. Mice with B cell-specific deficiency in IL35 subunits survive longer following an infection with the Salmonella bacteria than control mice. This enhanced infection control was associated with increased macrophage activation and Th1 responses $[53,54]$.

\section{Conclusion}

The role of IL-35 in asthma is still not well established. Despite a growing body of evidence showing that IL-35 is an important regulator of inflammatory cell infiltration, activation, and clearance in experimental models, limited data are available regarding the levels and functions of IL-35 in human asthma, and published studies have provided contradicting results. These contradictions in experimental results might be explained by the heterogeneity of airway inflammation in asthma and differences in the subtypes of asthma studied. Thus, additional studies with carefully phenotyped asthmatic patients are required to fully characterize the expression of IL-35 in the blood of asthmatic patients.

Recent studies using a number of different murine models of asthma indicate that IL-35 exerts anti-inflammatory effects in vivo. It suppresses allergen-induced AHR and inflammation. Targeting the actions of IL-35 might therefore present a promising strategy for the development of effective therapeutic agents against asthma.

\section{Disclosure}

The roles of the funding body were involved in the design of the study and writing the manuscript.

\section{Conflicts of Interest}

All authors have no conflicts of interest. There are no ethical/ legal conflicts involved in the article.

\section{Authors' Contributions}

Jie Zhang contributed to the conception of the study. Zhenzhong Su and Xuejiao Lv contributed significantly to the analysis and manuscript preparation. Peng Gao performed the data analyses and wrote the manuscript. All authors have read and approved the content.

\section{Acknowledgments}

This work was funded by the Jilin Province Science and Technology Development Plan Item (20150311066YY and 20160101135JC).

\section{References}

[1] L. W. Collison, V. Chaturvedi, A. L. Henderson et al., "IL-35mediated induction of a potent regulatory T cell population," Nature Immunology, vol. 11, no. 12, pp. 1093-1101, 2010.

[2] L. W. Collison, G. M. Delgoffe, C. S. Guy et al., "The composition and signaling of the IL-35 receptor are unconventional," Nature Immunology, vol. 13, no. 3, pp. 290-299, 2012.

[3] O. Devergne, M. Birkenbach, and E. Kieff, "Epstein-Barr virusinduced gene 3 and the p35 subunit of interleukin 12 form a novel heterodimeric hematopoietin," Proceedings of the National Academy of Sciences of the United States of America, vol. 94, no. 22, pp. 12041-12046, 1997.

[4] R. M. Pope and S. Shahrara, "Possible roles of IL-12-family cytokines in rheumatoid arthritis," Nature Reviews. Rheumatology, vol. 9, no. 4, pp. 252-256, 2013.

[5] D. H. Presky, H. Yang, L. J. Minetti et al., "A functional interleukin 12 receptor complex is composed of two beta-type cytokine receptor subunits," Proceedings of the National Academy of Sciences of the United States of America, vol. 93, no. 24, pp. 14002-14007, 1996.

[6] U. Grohmann, M. L. Belladonna, R. Bianchi et al., "IL-12 acts directly on DC to promote nuclear localization of NF-kappaB and primes DC for IL-12 production," Immunity, vol. 9, no. 3, pp. 315-323, 1998.

[7] W. Niedbala, X. Q. Wei, B. Cai et al., "IL-35 is a novel cytokine with therapeutic effects against collagen-induced arthritis through the expansion of regulatory $\mathrm{T}$ cells and suppression of Th17 cells," European Journal of Immunology, vol. 37, no. 11, pp. 3021-3029, 2007.

[8] X. Sha, S. Meng, X. Li et al., "Interleukin-35 inhibits endothelial cell activation by suppressing MAPK-AP-1 pathway," The Journal of Biological Chemistry, vol. 290, no. 31, pp. 1930719318, 2015.

[9] S. Wirtz, U. Billmeier, T. Mchedlidze, R. S. Blumberg, and M. F. Neurath, "Interleukin-35 mediates mucosal immune responses that protect against T-cell-dependent colitis," Gastroenterology, vol. 141, no. 5, pp. 1875-1886, 2011.

[10] K. Guttek and D. Reinhold, "Stimulated human peripheral T cells produce high amounts of IL-35 protein in a proliferation-dependent manner," Cytokine, vol. 64, no. 1, pp. 46-50, 2013.

[11] J. Q. Liu, Z. Liu, X. Zhang et al., "Increased Th17 and regulatory $\mathrm{T}$ cell responses in $\mathrm{EBV}$-induced gene 3-deficient mice lead to marginally enhanced development of autoimmune encephalomyelitis," Journal of Immunology (Baltimore, Md.: 1950), vol. 188, no. 7, pp. 3099-3106, 2012. 
[12] J. Yang, M. Yang, T. M. Htut et al., "Epstein-Barr virusinduced gene 3 negatively regulates IL-17, IL-22 and RORgamma t," European Journal of Immunology, vol. 38, no. 5, pp. 1204-1214, 2008.

[13] G. S. Whitehead, R. H. Wilson, K. Nakano, L. H. Burch, H. Nakano, and D. N. Cook, "IL-35 production by inducible costimulator (ICOS)-positive regulatory $\mathrm{T}$ cells reverses established IL-17-dependent allergic airways disease," The Journal of Allergy and Clinical Immunology, vol. 129, no. 1, pp. 207215, 2012, e201-205.

[14] L. W. Collison, C. J. Workman, T. T. Kuo et al., "The inhibitory cytokine IL-35 contributes to regulatory T-cell function," Nature, vol. 450, no. 7169, pp. 566-569, 2007.

[15] D. A. Vignali and V. K. Kuchroo, "IL-12 family cytokines: immunological playmakers," Nature Immunology, vol. 13, no. 8, pp. 722-728, 2012.

[16] I. Kochetkova, S. Golden, K. Holderness, G. Callis, and D. W. Pascual, "IL-35 stimulation of CD39+ regulatory T cells confers protection against collagen II-induced arthritis via the production of IL-10," Journal of Immunology (Baltimore, Md.: 1950), vol. 184, no. 12, pp. 7144-7153, 2010.

[17] M. Bettini, A. H. Castellaw, G. P. Lennon, A. R. Burton, and D. A. Vignali, "Prevention of autoimmune diabetes by ectopic pancreatic beta-cell expression of interleukin-35," Diabetes, vol. 61, no. 6, pp. 1519-1526, 2012.

[18] C. H. Huang, E. X. Loo, I. C. Kuo et al., "Airway inflammation and IgE production induced by dust mite allergen-specific memory/effector Th2 cell line can be effectively attenuated by IL-35," Journal of Immunology (Baltimore, Md. : 1950), vol. 187, no. 1, pp. 462-471, 2011.

[19] A. Thiolat, A. Denys, M. Petit et al., "Interleukin-35 gene therapy exacerbates experimental rheumatoid arthritis in mice," Cytokine, vol. 69, no. 1, pp. 87-93, 2014.

[20] Y. Li, Y. Wang, Y. Liu et al., "The possible role of the novel cytokines il-35 and il-37 in inflammatory bowel disease," Mediators of Inflammation, vol. 2014, Article ID 136329, p. 10, 2014.

[21] Y. Lin, Y. Huang, Z. Lu et al., "Decreased plasma IL-35 levels are related to the left ventricular ejection fraction in coronary artery diseases," PloS One, vol. 7, no. 12, article e52490, 2012.

[22] P. Jin, H. Ren, W. Sun, W. Xin, H. Zhang, and J. Hao, "Circulating IL-35 in pancreatic ductal adenocarcinoma patients," Human Immunology, vol. 75, no. 1, pp. 29-33, 2014.

[23] X. Gu, T. Tian, B. Zhang et al., "Elevated plasma interleukin-35 levels predict poor prognosis in patients with non-small cell lung cancer," Tumour Biology: The Journal of the International Society for Oncodevelopmental Biology and Medicine, vol. 36, no. 4, pp. 2651-2656, 2015.

[24] Y. L. Zhang, X. Y. Zhou, X. Y. Guo, and J. W. Tu, "Association between serum interleukin-35 levels and severity of acute pancreatitis," International Journal of Clinical and Experimental Medicine, vol. 8, no. 5, pp. 7430-7434, 2015.

[25] C. Y. Yue, B. Zhang, and C. M. Ying, "Elevated serum level of IL-35 associated with the maintenance of maternal-fetal immune tolerance in normal pregnancy," PloS One, vol. 10, no. 6, article e0128219, 2015.

[26] M. Tomcik, P. Zerr, K. Palumbo-Zerr et al., "Interleukin-35 is upregulated in systemic sclerosis and its serum levels are associated with early disease," Rheumatology (Oxford, England), vol. 54, no. 12, pp. 2273-2282, 2015.

[27] C. K. Wong, T. F. Leung, I. M. Chu, J. Dong, Y. Y. Lam, and C. W. Lam, "Aberrant expression of regulatory cytokine
IL-35 and pattern recognition receptor NOD2 in patients with allergic asthma," Inflammation, vol. 38, no. 1, pp. 348-360, 2015.

[28] W. Wang, P. Li, and J. Yang, "Decreased circulating interleukin-35 levels are related to interleukin-4-producing CD8+ T cells in patients with allergic asthma," Iranian Journal of Allergy, Asthma, and Immunology, vol. 14, no. 4, pp. 379$385,2015$.

[29] E. Bateman, S. S. Hurd, P. J. Barnes et al., "Global strategy for asthma management and prevention: GINA executive summary," The European Respiratory Journal, vol. 31, no. 1, pp. 143-178, 2008.

[30] A. B. Kay, "The role of T lymphocytes in asthma," Chemical Immunology and Allergy, vol. 91, pp. 59-75, 2006.

[31] W. W. Busse and R. F. Lemanske Jr., "Asthma," The New England Journal of Medicine, vol. 344, no. 5, pp. 350-362, 2001.

[32] J. L. Simpson, R. Scott, M. J. Boyle, and P. G. Gibson, "Inflammatory subtypes in asthma: assessment and identification using induced sputum," Respirology (Carlton, Vic.), vol. 11, no. 1, pp. 54-61, 2006.

[33] P. Haldar, C. E. Brightling, B. Hargadon et al., "Mepolizumab and exacerbations of refractory eosinophilic asthma," The New England Journal of Medicine, vol. 360, no. 10, pp. 973-984, 2009.

[34] P. J. Barnes, "Immunology of asthma and chronic obstructive pulmonary disease," Nature Reviews. Immunology, vol. 8, no. 3, pp. 183-192, 2008.

[35] P. G. Woodruff, B. Modrek, D. F. Choy et al., "T-helper type 2driven inflammation defines major subphenotypes of asthma," American Journal of Respiratory and Critical Care Medicine, vol. 180, no. 5, pp. 388-395, 2009.

[36] E. Mortaz, M. Reza Masjedi, A. Allameh, and I. M Adcock, "Inflammasome signaling in pathogenesis of lung diseases," Current Pharmaceutical Design, vol. 18, no. 16, pp. 2320 2328, 2012.

[37] G. dos Santos, M. A. Kutuzov, and K. M. Ridge, "The inflammasome in lung diseases," American Journal of Physiology. Lung Cellular and Molecular Physiology, vol. 303, no. 8, pp. L627-L633, 2012.

[38] P. W. Hellings, A. Kasran, Z. Liu et al., "Interleukin-17 orchestrates the granulocyte influx into airways after allergen inhalation in a mouse model of allergic asthma," American Journal of Respiratory Cell and Molecular Biology, vol. 28, no. 1, pp. 4250, 2003.

[39] L. McKinley, J. F. Alcorn, A. Peterson et al., "TH17 cells mediate steroid-resistant airway inflammation and airway hyperresponsiveness in mice," Journal of Immunology (Baltimore, Md.: 1950), vol. 181, no. 6, pp. 4089-4097, 2008.

[40] R. H. Wilson, G. S. Whitehead, H. Nakano, M. E. Free, J. K. Kolls, and D. N. Cook, "Allergic sensitization through the airway primes Th17-dependent neutrophilia and airway hyperresponsiveness," American Journal of Respiratory and Critical Care Medicine, vol. 180, no. 8, pp. 720-730, 2009.

[41] A. Barczyk, W. Pierzchala, and E. Sozanska, "Interleukin-17 in sputum correlates with airway hyperresponsiveness to methacholine," Respiratory Medicine, vol. 97, no. 6, pp. 726-733, 2003.

[42] H. Wakashin, K. Hirose, Y. Maezawa et al., "IL-23 and Th17 cells enhance Th2-cell-mediated eosinophilic airway inflammation in mice," American Journal of Respiratory and Critical Care Medicine, vol. 178, no. 10, pp. 1023-1032, 2008. 
[43] H. von Boehmer, "Mechanisms of suppression by suppressor T cells," Nature Immunology, vol. 6, no. 4, pp. 338-344, 2005.

[44] C. Dejaco, C. Duftner, B. Grubeck-Loebenstein, and M. Schirmer, "Imbalance of regulatory T cells in human autoimmune diseases," Immunology, vol. 117, no. 3, pp. 289-300, 2006.

[45] M. Möttönen, J. Heikkinen, L. Mustonen, P. Isomäki, R. Luukkainen, and O. Lassila, "CD4+ CD25+ T cells with the phenotypic and functional characteristics of regulatory $\mathrm{T}$ cells are enriched in the synovial fluid of patients with rheumatoid arthritis," Clinical and Experimental Immunology, vol. 140, no. 2, pp. 360-367, 2005.

[46] H. Ait-Oufella, B. L. Salomon, S. Potteaux et al., "Natural regulatory $\mathrm{T}$ cells control the development of atherosclerosis in mice," Nature Medicine, vol. 12, no. 2, pp. 178-180, 2006.

[47] C. M. Lloyd and C. M. Hawrylowicz, "Regulatory T cells in asthma," Immunity, vol. 31, no. 3, pp. 438-449, 2009.

[48] M. Akdis, J. Verhagen, A. Taylor et al., "Immune responses in healthy and allergic individuals are characterized by a fine balance between allergen-specific T regulatory 1 and T helper 2 cells," The Journal of Experimental Medicine, vol. 199, no. 11, pp. 1567-1575, 2004.

[49] S. H. Lee, S. Goswami, A. Grudo et al., "Antielastin autoimmunity in tobacco smoking-induced emphysema," Nature Medicine, vol. 13, no. 5, pp. 567-569, 2007.

[50] J. Bai, S. L. Qiu, X. N. Zhong et al., "Erythromycin enhances CD4+Foxp3+ regulatory T-cell responses in a rat model of smoke-induced lung inflammation," Mediators of Inflammation, vol. 2012, Article ID 410232, p. 9, 2012.

[51] Y. Li, X. Pan, X. Peng et al., "Adenovirus-mediated interleukin-35 gene transfer suppresses allergic airway inflammation in a murine model of asthma," Inflammation Research, vol. 64, no. 10, pp. 767-774, 2015.

[52] J. Dong, C. K. Wong, Z. Cai, D. Jiao, M. Chu, and C. W. Lam, "Amelioration of allergic airway inflammation in mice by regulatory IL-35 through dampening inflammatory dendritic cells," Allergy, vol. 70, no. 8, pp. 921-932, 2015.

[53] W. van de Veen, B. Stanic, O. F. Wirz, K. Jansen, A. Globinska, and M. Akdis, "Role of regulatory B cells in immune tolerance to allergens and beyond," The Journal of Allergy and Clinical Immunology, vol. 138, no. 3, pp. 654-665, 2016.

[54] P. Shen, T. Roch, V. Lampropoulou et al., "IL-35-producing B cells are critical regulators of immunity during autoimmune and infectious diseases," Nature, vol. 507, no. 7492, pp. 366370, 2014. 


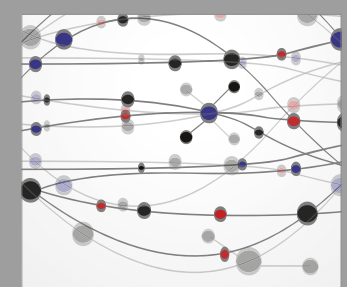

The Scientific World Journal
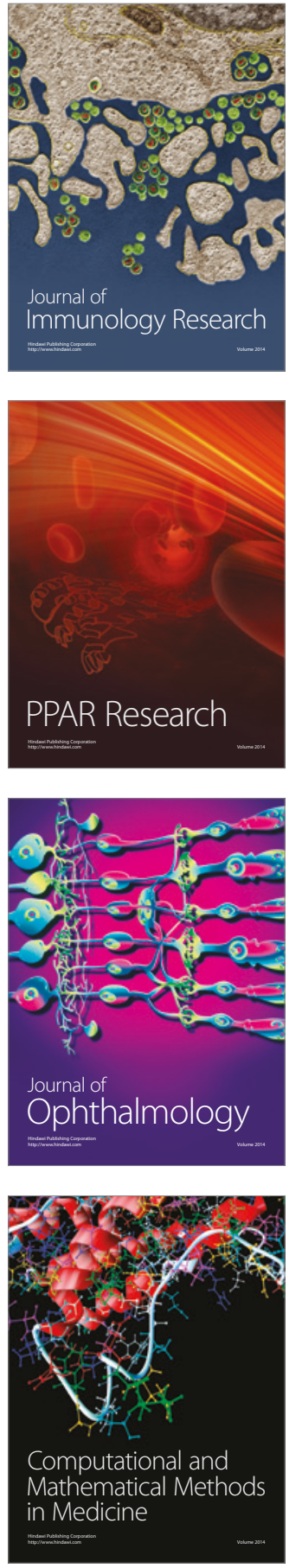

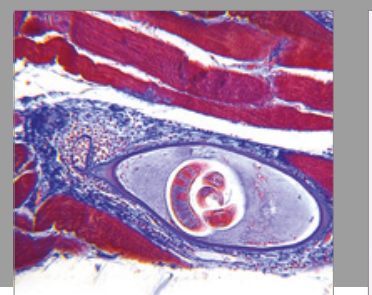

Gastroenterology Research and Practice
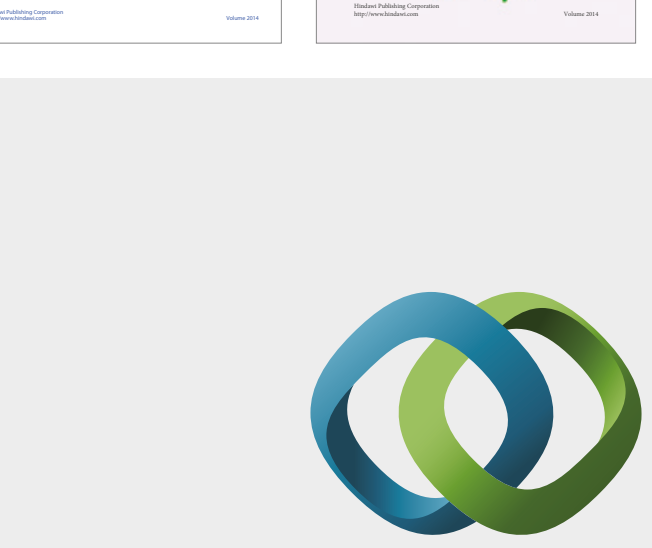

\section{Hindawi}

Submit your manuscripts at

https://www.hindawi.com
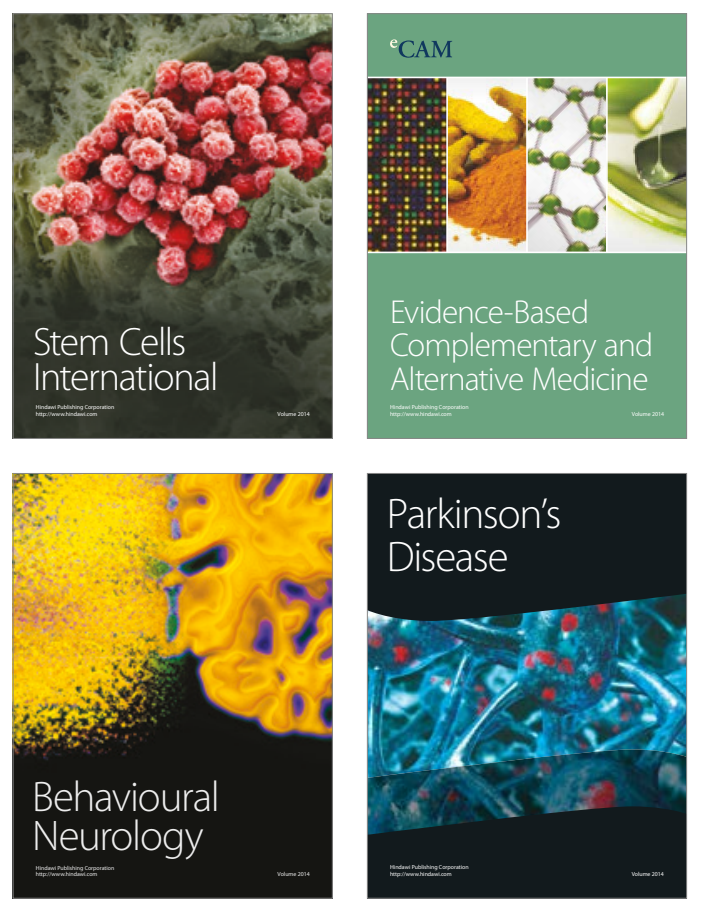
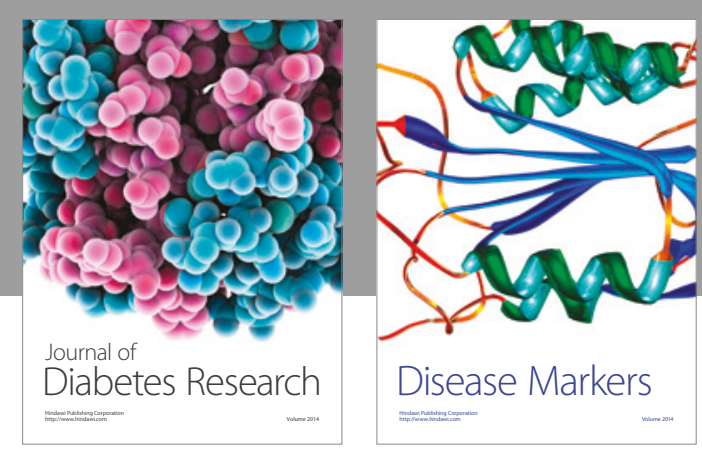

Disease Markers
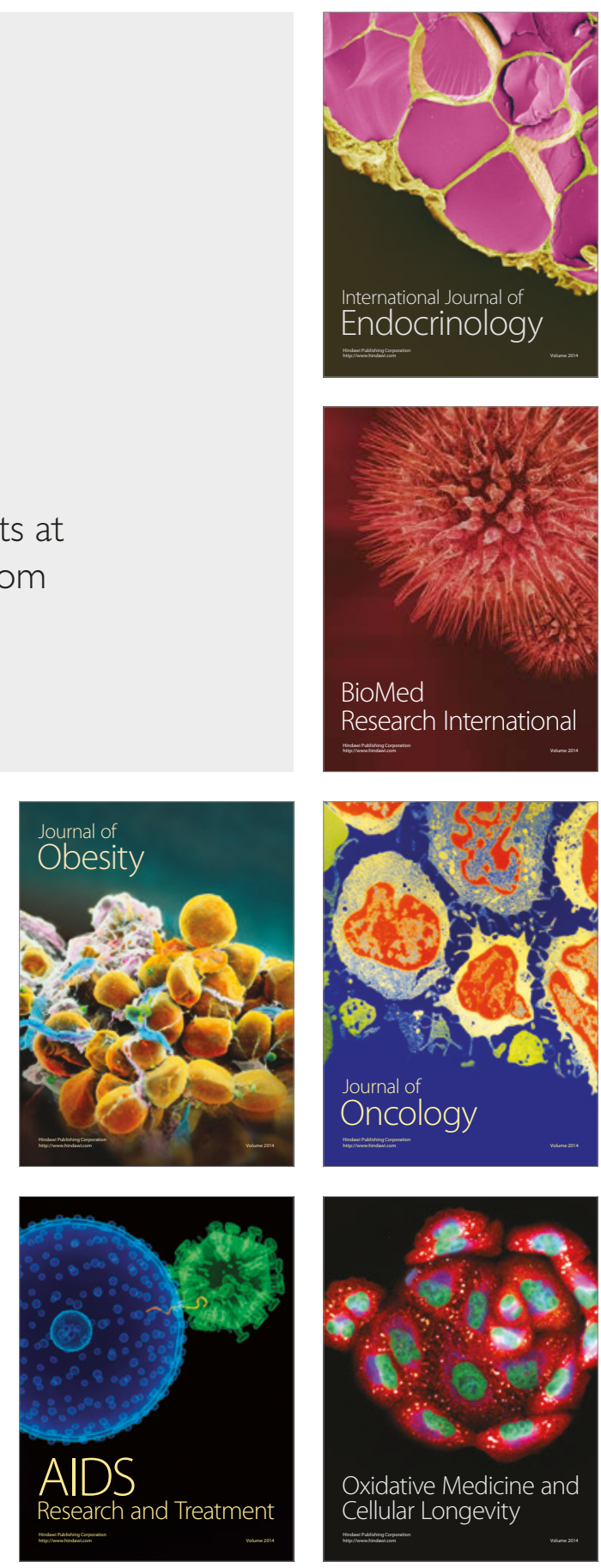